\title{
Analysis of Surface Plasmon Polariton Conversion Coefficient In Slit-Groove Structure
}

Yeonsang Park ( $\square$ yeonsang.park@cnu.ac.kr)

Chungnam National University https://orcid.org/0000-0002-9746-8026

\section{Research Article}

Keywords: surface plasmon polariton, conversion coefficient, slit-groove structure, scattering intensities, FDTD simulation

Posted Date: July 29th, 2021

DOl: https://doi.org/10.21203/rs.3.rs-726773/v1

License: (9) This work is licensed under a Creative Commons Attribution 4.0 International License. Read Full License

Version of Record: A version of this preprint was published at Journal of the Korean Physical Society on February 10th, 2022. See the published version at https://doi.org/10.1007/s40042-022-00419-9. 


\section{Abstract}

A method for the analysis of finding the conversion efficiency of the surface plasmon polariton in slitgroove structures is proposed and studied. The conversion efficiency of the surface plasmon polariton can be determined by measuring the intensities of light scattered at the slit and groove positions. To verify the usefulness of the proposed method, two rigorous simulations based on the finite-difference time-domain method were executed and the simulation results compared with previously reported data. One was to mimic the far-field imaging experiment in slit-groove structure and the other was to calculate the conversion coefficient directly in a single scattering structure. The SPP conversion efficiencies obtained from the two simulations were approximately 0.232 and 0.220 respectively, and these agreed with the reported data. The suggested method can be used regardless of the shape of the plasmongeneration structure; therefore, it is expected to be useful in a wide range of experiments with different scattering structures.

\section{Introduction}

Since the discovery of surface plasmon polaritons (SPPs), researchers have paid significant attention to them because they enable sub-wavelength focusing and filed enhancement in nanophotonics [1, 2], as well as their potential applications to light emission control and plasmonic integrated circuits [3, 4]. SPPs are promising candidates for integrated photonic circuits in sub-micrometer scale because they can be confined and propagate in the sub-wavelength scale [5]. Therefore, they have great potential to bridge electronics and photonics that face the difficulty in integrating photonic circuits with electronic chips because of the size mismatch problem [6]. To launch SPPs on planar chips, a structure providing momentum transfer is essential because of the mismatch of the dispersion curve between light and SPPs. Momentum delivery can be realized either by evanescent coupling via total internal reflection (Kretschmann geometry) or coupling into a thin narrow slit [7]. Among these, the latter is easy to fabricate on planar metal surfaces and also efficient for SPP creation. A slit or groove edge provides additional momentum to the incident light so that the dispersion of light can be matched by the dispersion curve of SPPs. Therefore, incident light can be converted into SPPs at the interface of the metal and dielectric layers [8].

Since SPPs are intrinsically near-field waves, they cannot be measured by conventional optics measuring far-field intensity of light. Traditional optical components can detect the intensity of only radiating and not near-field waves that decay exponentially. Therefore, probe-detecting apparatus such as near-field scanning optical microscopes (NSOMs) are necessary to measure SPPs [9]. From images of SPPs obtained by NSOMs, it is not easy to determine the coupling efficiency of SPPS with a slit structure [1012]. To overcome this difficulty, an easy method to determine the coupling efficiency of SPPs by using intensities obtained in far-field measurements is presented and explained. To check the validity of the proposed method in this study, finite-difference time-domain (FDTD) simulations were performed [13] and the obtained conversion efficiencies compared with reference data. Two different FDTD simulations were executed to extract the SPP conversion efficiency of the slit structure. One was to mimic a real experiment 
of a far-field imaging experiment, which can be called a virtual experiment, and the other was a direct calculation of the SPP conversion efficiency in a single-slit structure.

\section{Experiments And Discussion}

\section{Analysis of SPP conversion efficiency}

To extract the conversion efficiency of SPPs in far-field imaging measurements, a slit-groove structure for SPP generation and scattering measurements was used [14, 15]. A schematic of the slit-groove structure is shown in Fig. 1. A transmission measurement setup was used. The metal for the SPPs was deposited on the substrate, and it is assumed that the metal film is thick enough to not transmit the incident light. By exposing the back of the sample to the incident light, SPPs can be launched by propagating toward the normal direction of the length of the slit with the aid of slit geometry. When light is incident from the substrate, some of the incident light (intensity $I_{0}$ ) is reflected and converted as SPPS at the bottom side of the substrate because of the edge of the slit structure. Additionally, light transmitting through the slit structure is absorbed by the metal film. If the coefficient of light reaching the top side of the substrate is written as $\gamma$, some of $\gamma I_{0}$ is also converted to SPPs at the top side of the substrate because it meets the edge structure of the slit. When the conversion coefficient of SPPs by the edge structure is denoted as $C_{S}$ the SPP-converted intensity of incident light at the top surface of the substrate can be written as $2 C_{s} Y I_{O}$ owing to two edge structures. Therefore, the intensity transmitted through the slit was measured by the detector located above the slit.

$I_{s}=I_{O} Y\left(1-2 C_{s}\right)$

Similarly, the intensity measured by the detector located above the groove structure can be analyzed as follows: SPPs generated by the top edge of the slit propagate in the direction normal to the slit length at the front metal interface. The initial intensity of the SPPs propagating toward the groove corresponded to $C_{s} Y I_{O}$. Then, SPPs are decayed by the $\exp (-a d)$ factor, while they propagate the distance of $d$ on the metal interface. The parameter of $a$ is the double of the imaginary part of the wavevector $\left(k_{s p p}=k_{n, s p p}+i k_{i, s p p}\right)$ of the SPP mode formed at the interface, and the propagation length of the SPPs at the interface of the metal and air can be denoted as $1 / a$. When the propagated SPPs meets a groove located at a distance $d$ from the slit, they scatter and convert into light because of the edge of the groove structure, as in the case of the slit. When the coefficient of which light is converted from SPPs to light and scattered by the edge of the groove is written as $C_{g}$, the intensity obtained at the groove position is as follows:

$$
I_{g}=I_{0} \gamma C_{s}\left|e^{i k_{s p p} d}\right|^{2} C_{g}=I_{0} \gamma C_{s}\left|e^{i\left(k_{n, s p p}+i k_{i, s p p}\right) d}\right|^{2} C_{g}=I_{0} \gamma C_{s}\left|e^{2 i k_{n, s p p} d}\right| e^{-2 k_{i, s p p} d} C_{g}=I_{0} \gamma C_{s} e^{-\alpha d} C_{g}
$$

At that time, the coefficient of light-to-plasmon conversion, $C_{s^{\prime}}$ and plasmon-to-light conversion, $C_{g^{\prime}}$ should be the same because the edge geometries of the slit and groove are the same. Therefore, Eq. (2) can be expressed as follows: 
$I_{g}=I_{0} \gamma C_{s}^{2} e^{-\alpha d}$

Finally, the SPP conversion coefficient can be obtained as Eq. (5) by solving the quadratic Eq. (4) related to the ratio of $I_{s}$ and $I_{g}$.

$$
C_{s}^{2}+2 M C_{s}-M=0 \quad \text { where } \quad M \equiv \frac{I_{g}}{I_{s}} e^{\alpha d}
$$

$$
C_{s}=\sqrt{M^{2}+M}-M
$$

In Eq. (5), the parameter $M$ can be extracted directly from the scattered intensities measured in the farfield imaging experiment. In particular, the decay parameter $a$ of the SPP propagation can be obtained from the propagation measurement experiment and simultaneously calculated by solving the dispersion of the SPP formed at the interface of the metal and air [16]. Additionally, it is worth noting that this analysis of the conversion coefficient does not depend on the shape of the scattering structure. Therefore, if the shapes of the scattering structure in both light-to-plasmon and plasmon-to-light conversions are the same, the suggested approach can be applied in any plasmon experiment irrespective of the geometry of scattering.

\section{Comparison of two FDTD simulations}

The FDTD simulation can solve Maxwell equations sequentially and directly in an interesting structure. Therefore, two types of FDTD simulations (Lumerical FDTD simulator) were executed. One mimicked the real experiment of measuring the far-field intensities and propagation decay length. From this simulation, the parameter $M$ involving slit scattering intensity, groove scattering intensity, and propagation decay length were extracted directly by analyzing the far-field images. The other simulation determined the SPP conversion coefficient of the slit structure directly by simulating only one slit structure.

Figure 2(a) shows a schematic of the slit-groove structure used in the first FDTD simulation. In this simulation, a gold layer with a thickness of $300 \mathrm{~nm}$ was used as the metal layer for the SPP generation, and the width of the slit was $150 \mathrm{~nm}$, the same as that of the groove. The depth of the groove was 150 $\mathrm{nm}$, and the wavelength of the incident light was $600 \mathrm{~nm}$. Detection monitors that measure scattering intensities in front of the sample and the propagation decay length in the simulation are also shown in the schematic. Monitor 1 , indicated by the black line, is located $1.2 \mu \mathrm{m}$ away from the top surface of the metal layer and measures scattering intensities by using the slit and groove. Monitor 2 , indicated by the gray dashed line, is located $250 \mathrm{~nm}$ away from the top surface of the metal layer and measures the intensity of the propagating SPP at the interface of the metal and air. From the results obtained by monitors 1 and 2, parameter $M$ can be extracted. When a real experiment is performed, $M$ can also be obtained by measuring the intensities scattered at the slit and groove in far-field imaging, and by fitting the propagation length in the intensity plot of SPP propagation as an exponential function of distance. Figure 2(b) shows the distribution of the electric field (E-field) intensity obtained in monitor 2 of the FDTD simulation. Figure 2(c) is an E-field distribution detected at monitor 3 normal to the $x$-direction located 1.5 
$\mu \mathrm{m}$ away from the slit structure. From Fig. 2(b), it is clear that the light converted into SPPs was scattered by the groove structure. Figure 3 shows the intensity plot obtained using monitor 2 . The scattering intensity of $I_{s}$ at the slit structure can be obtained by summing the whole E-field intensities in the left area enclosed by yellow color in Fig. 3. Similarly, the scattering intensity of $I_{g}$ at the groove structure could be extracted by summing the intensities in the right area enclosed by cyan color. In addition to $I_{s}$ and $I_{g}$, the parameter of $a$ could also be obtained from this plot by fitting the decay intensities of the plot as an exponential function of $x$, as shown by the red line in Fig. 3. As shown in Fig. 3, the decay parameter of $a$ was fitted as $~ 2.105$. Finally, the parameter $M$ in Eq. (4) was calculated as $\sim 1.768$, and the coupling coefficient of the SPP conversion was obtained as $\sim 0.232$ from Eq. (5). By comparing the obtained number with those previously reported, it is clear that the coupling efficiency obtained from this simulation agreed with values previously reported in some literatures, thus the proposed analysis method is useful $[10,11,17-22]$.

The second FDTD simulation was executed to verify the usefulness of the suggested analysis. In this case, the coupling coefficient was extracted directly by simulating a single-slit structure. Figure 4(a) shows the schematic of the single-slit structure used in the second FDTD simulation. For the single-slit simulation, a gold layer with a thickness of $300 \mathrm{~nm}$ and a slit with a width of $150 \mathrm{~nm}$ were used. In addition, the wavelength of the incident light was $600 \mathrm{~nm}$. The schematic shows detection monitors enclosing the slit structure in the analysis. In a single-slit structure, the incident light from the substrate is divided into four parts. The incident light is reflected (reflection $R$ ), transmitted (transmission $T$ ), absorbed (absorption $A$ ), and converted into SPPs at four edges of the slit structure (conversion $C_{S}$ ). $R$ and $T$ can be calculated from Monitors 1 and 2, which are located apart by $1.2 \mu \mathrm{m}$ away from the metal layer. The conversion coefficient of $C_{s}$ can be extracted by multiplying the intensity of monitor 3 or 4 normal to the $x$ direction, as shown in Fig. 4 , by the factor of $\exp (\beta s)$. Here, $s$ is the distance of the monitoring location in the $x$-direction and $\beta$ is the decay parameter of the SPPs. Since SPPs generated at the slit decay because of absorption by the metal, the intensity $I_{S P P}$ measured by the monitor apart by $s$ should be compensated by multiplying the factor of $\exp (\beta s)$. Therefore, $C_{S}$ can be calculated from $I_{S P P^{\bullet}} \exp (\beta S)$. The absorption $A$ can also be extracted from the simulation by calculating the boxed region that is shown by the dashed gray box in Fig. 4(a). The absorption at the slit structure can be obtained by directly calculating the absorption power of $P_{a b s}=-0.5 \mathrm{real}\left((\nabla \bullet P)=-0.5 \mathrm{\omega}|\mathrm{E}|^{2} \operatorname{imag}(\varepsilon)\right.$ from simulated E-fields in monitor 3 [23]. Figure $4(b)$ also shows the bound SPP mode supported by the metal layer and air, which was obtained by monitor 3 located at the position apart by $1.5 \mu \mathrm{m}$ from the slit structure. Figure 4(c) shows the spectra of the $T$ and $C_{s}$ obtained from the simulation. The calculated $C_{s}$ was $\sim 0.220$, which is close to the result obtained from the first slit-groove simulation and the previously reported data. This also verified the validity of the suggested analysis. Two conversion coefficient $C s$ values calculated from two FDTD simulations and the data reported in some references are listed in Table 1 for the comparison. 
Table 1

Coefficients of SPP conversion extracted from slit-groove simulation, single slit simulation, and reference data.

\begin{tabular}{|c|lllll|}
\hline & \multicolumn{4}{l}{ FDTD simulation } & \multicolumn{3}{l|}{ Reported experimental data } \\
\cline { 2 - 6 } & slit-groove & single slit & Ref. 18, 19 & Ref. 20 & Ref. 22 \\
\hline$C_{s}$ & $\sim 0.232$ & $\sim 0.220$ & $\sim 0.38$ & $\sim 0.20$ & $\sim 0.28$ \\
\hline
\end{tabular}

\section{Conclusion}

In conclusion, a method for obtaining the SPP conversion efficiency from a slit-groove imaging experiment was proposed and verified through FDTD simulations. By matching the parameters measured in the far-field imaging simulation with those obtained from the proposed analysis, the SPP conversion efficiency was obtained independent of the shape of the scattering structure. FDTD simulations with two different approaches were performed to verify the proposed analysis: one to mimic the entire far-field imaging experiment by using FDTD simulation and the other to calculate the conversion coefficient directly in a single scattering structure. The SPP conversion efficiencies obtained from the two simulations were approximately 0.232 and 0.220 , respectively, in close agreement with each other. From this agreement, the usefulness of the suggested analysis was verified, and it is expected to be applicable in various plasmon experiments because the analysis is independent of the shape of the plasmon scattering structure.

\section{Declarations}

Funding: Not applicable

Conflicts of interest/Competing interests: Not applicable

Availability of data and material: Not applicable

Code availability: Lumerical FDTD simulator

Author's contribution: Y.P. conceived the idea and performed simulations. Y.P wrote the manuscript.

Ethics approval: Not applicable

Consent to participate: Not applicable

Consent to publication: Not applicable

\section{ACKNOWLEDGMENTS}

This work was supported by funding from the Chungnam National University. 


\section{References}

1. Ebbesen TW, Lezec HJ, Ghaemi HF, Thio T, Wolff PA (1998) Extraordinary optical transmission through sub-wavelength hole arrays. Nature 391:667-669. https://doi.org/10.1038/35570

2. Ditlbacher H, Krenn JR, Hohenau A, Leitner A, Aussenegg FR (2003) Efficiency of local light-plasmon coupling. Appl Phys Lett 83:3665-3667. https://doi.org/10.1063/1.1625107

3. McNab SJ, Moll N, Vlasov YYA (2003) Ultra-low loss photonic integrated circuit with membrane-type photonic crystal waveguides. Opt Express 11:2927-2939. https://doi.org/10.1364/OE.11.002927

4. Polman A (2008) Applied physics. Plasmonics applied. Science 322:868-869. https://doi.org/10.1126/science.1163959

5. Barnes WL, Dereux A, Ebbesen TW (2003) Surface plasmon subwavelength optics. Nature 424:824830. https://doi.org/10.1038/nature01937

6. Ozbay E (2006) Plasmonics: merging photonics and electronics at nanoscale dimensions. Science 311:189-193. https://doi.org/10.1126/science.1114849

7. Raether H (1998) Surface plasmons on smooth surfaces. In: Surface plasmons on smooth and rough surfaces and on gratings. Springer

8. Teng YY, Stern EA (1967) Plasma radiation from metal grating surfaces. Phys Rev Lett 19:511-514. https://doi.org/10.1103/PhysRevLett.19.511

9. Falk AL, Koppens FHL, Yu CL, Kang K, de Leon Snapp N, Akimov AV, Jo M, Lukin MD, Park H (2009) Near-field electrical detection of optical plasmons and single-plasmon sources. Nat Phys 5:475-479. https://doi.org/10.1038/nphys1284

10. Aigouy L, Lalanne P, Hugonin JP, Julié G, Mathet V, Mortier M (2007) Near-field analysis of surface waves launched at nanoslit apertures. Phys Rev Lett 98:153902.

https://doi.org/10.1103/PhysRevLett.98.153902

11. Iqbal T (2017) Coupling efficiency of surface plasmon polaritons: far- and near-field analyses. Plasmonics 12:215-221. https://doi.org/10.1007/s11468-016-0252-z

12. Sierant A, Jany BR, Kawalec T (2021) Near-field characterization of surface plasmon polaritons on a nanofabricated transmission structure. Phys Rev B 103.

https://doi.org/10.1103/PhysRevB.103.165433, 165433

13. Taflove A, Hagness SC, Piket-May M (2005) Computational electromagnetics: the finite-difference time-domain method. The Electrical Engineering Handbook

14. Pacifici D, Lezec HJ, Atwater HA (2007) All-optical modulation by plasmonic excitation of CdSe quantum dots. Nat Photonics 1:402-406. https://doi.org/10.1038/nphoton.2007.95

15. Morrill D, Li D, Pacifici D (2016) Measuring subwavelength spatial coherence with plasmonic interferometry. Nat Photonics 10:681-687. https://doi.org/10.1038/nphoton.2016.162

16. Neogi A, Lee C, Everitt HO, Kuroda T, Tackeuchi A, Yablonovitch E (2002) Enhancement of spontaneous recombination rate in a quantum well by resonant surface plasmon coupling. Phys Rev B 66. https://doi.org/10.1103/PhysRevB.66.153305, 153305 
17. Wang $B$, Lalanne $P$ (2010) How many surface plasmons are locally excited on the ridges of metallic lamellar gratings? Appl Phys Lett 96:051115. https://doi.org/10.1063/1.3304021

18. Lalanne P, Hugonin JP, Rodier JC (2005) Theory of surface plasmon generation at nanoslit apertures. Phys Rev Lett 95:263902. https://doi.org/10.1103/PhysRevLett.95.263902

19. Lalanne P, Hugonin JP, Rodier JC (2006) Approximate model for surface-plasmon generation at slit apertures. J Opt Soc Am A Opt Image Sci Vis 23:1608-1615.

https://doi.org/10.1364/JOSAA.23.001608

20. Mehfuz R, Maqsood MW, Chau KJ (2010) Enhancing the efficiency of slit-coupling to surfaceplasmon-polaritons via dispersion engineering. Opt Express 18:18206-18216. https://doi.org/10.1364/OE.18.018206

21. Hu H, Zeng X, Wang L, Xu Y, Song G, Gan Q (2012) Surface plasmon coupling efficiency from nanoslit apertures to metal-insulator-metal waveguides. Appl Phys Lett 101. https://doi.org/10.1063/1.4754137, 121112

22. Baudrion AL, de Léon-Pérez F, Mahboub 0 , Hohenau A, Ditlbacher H, García-Vidal FJ, Dintinger J, Ebbesen TW, Martin-Moreno L, Krenn JR (2008) Coupling efficiency of light to surface plasmon polariton for single subwavelength holes in a gold film. Opt Express 16:3420-3429. https://doi.org/10.1364/OE.16.003420

23. Jackson JD (1999) Classical electrodynamics. American Association of Physics Teachers

\section{Figures}

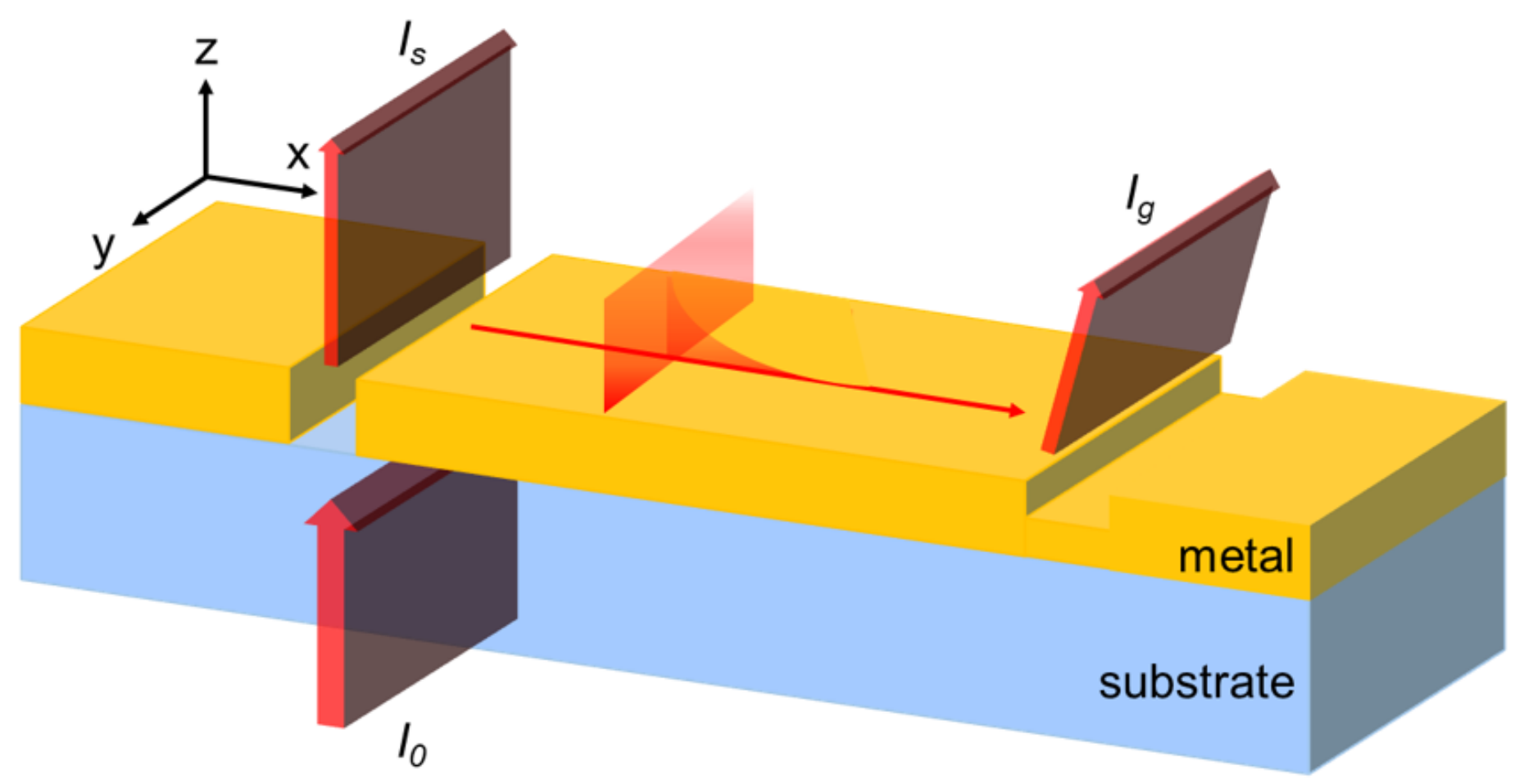


(a) monitor1

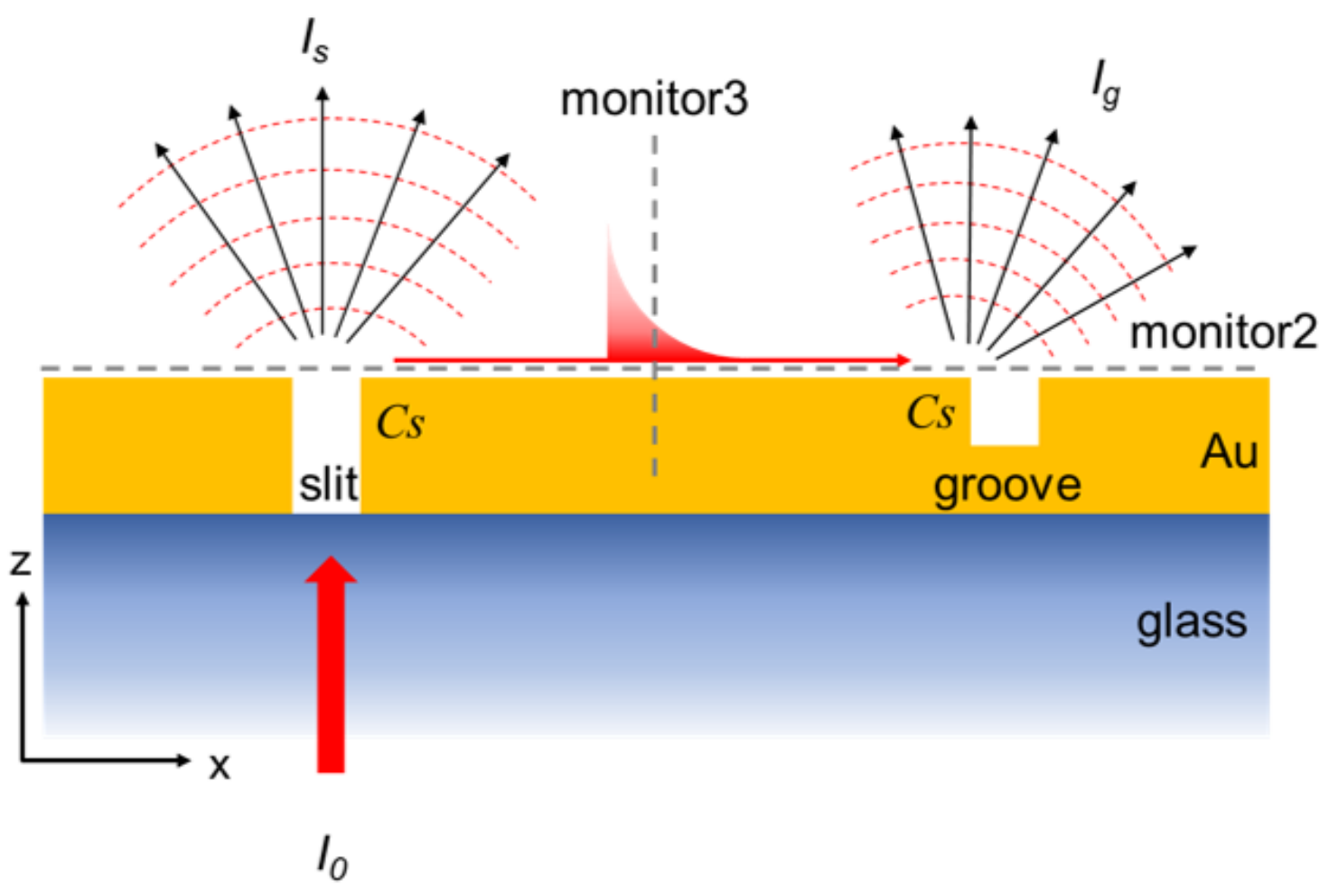

(b)

(c)
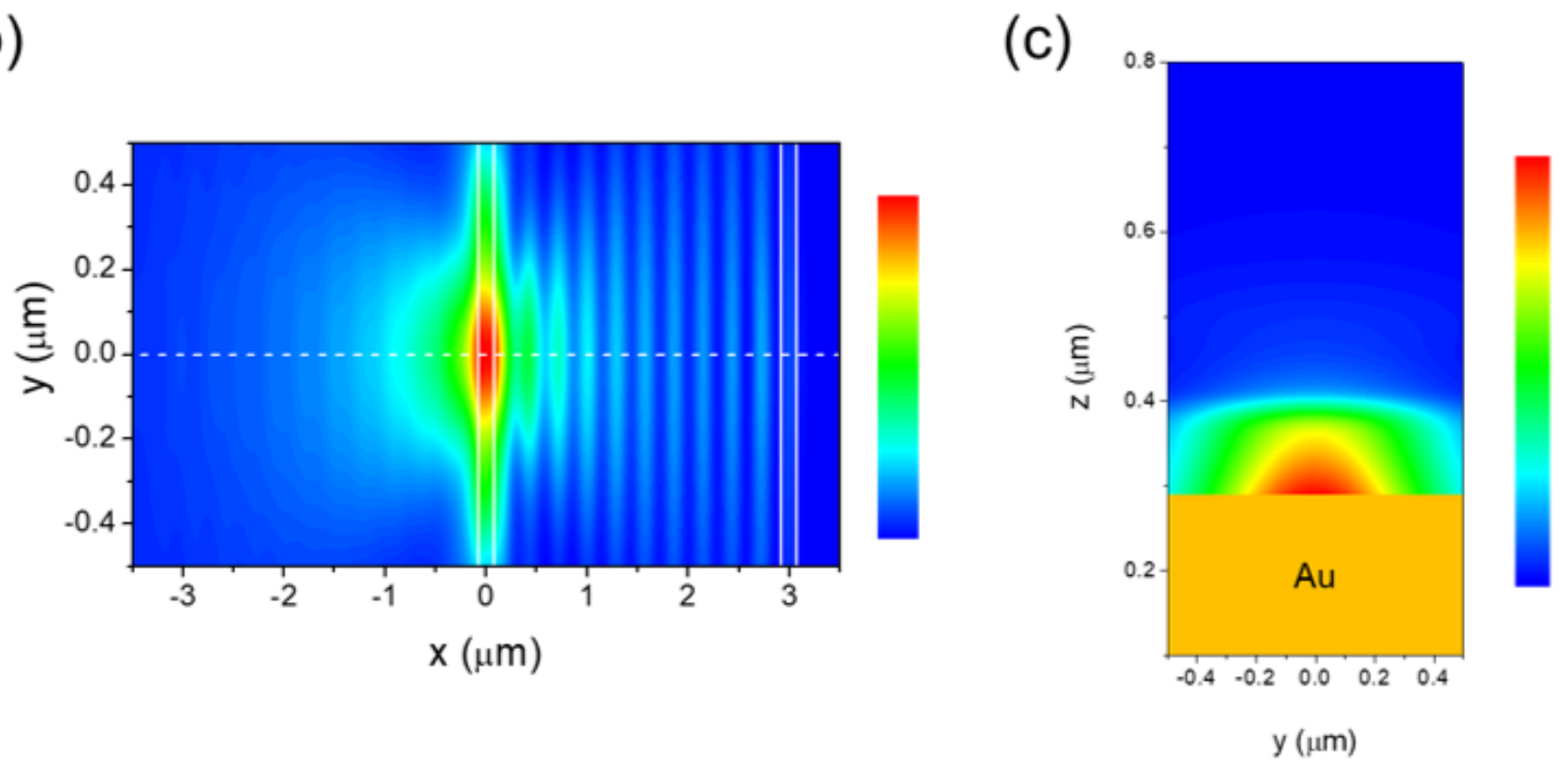

Figure 2

(a) Schematic of slit-groove structure used in FDTD simulations. Black line represents the position of monitor 1, which measured the scattering intensities. The two dashed gray lines indicate monitors 2 and 3. Monitor 2 detects SPP propagation at the top of the metal layer for the extraction of the propagation decay length $a$, and monitor 3 normal to $x$ direction detects E-field of SPPs bound to the metal surface. 
(b) Simulated E-field intensity obtained by monitor 2 located $450 \mathrm{~nm}$ away from the top surface of metal layer. (c) Simulated E-field intensity obtained by monitor 3 located $1.5 \mu \mathrm{m}$ away from the slit structure representing the SPP mode at the top surface of metal layer

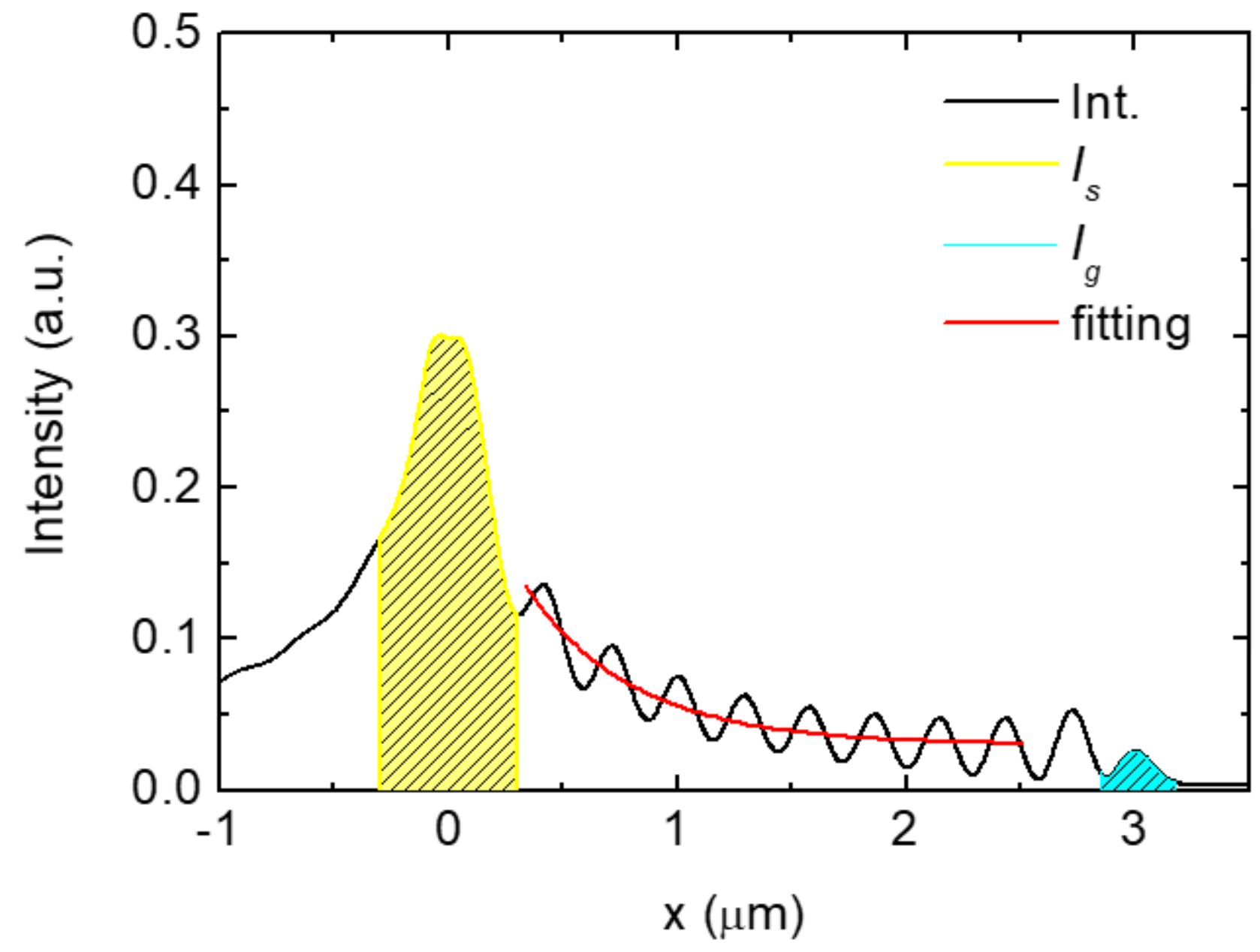

Figure 3

Calculation of scattering intensities Is and Ig and propagation decay length $\mathrm{a}$. The black line shows the intensity plot of SPP mode propagating to the groove structure. The yellow and cyan dashed regions correspond to Is and Ig respectively. The red line is the fitted curve of the intensity as a function of propagation distance 
(a)

\section{monitor1}

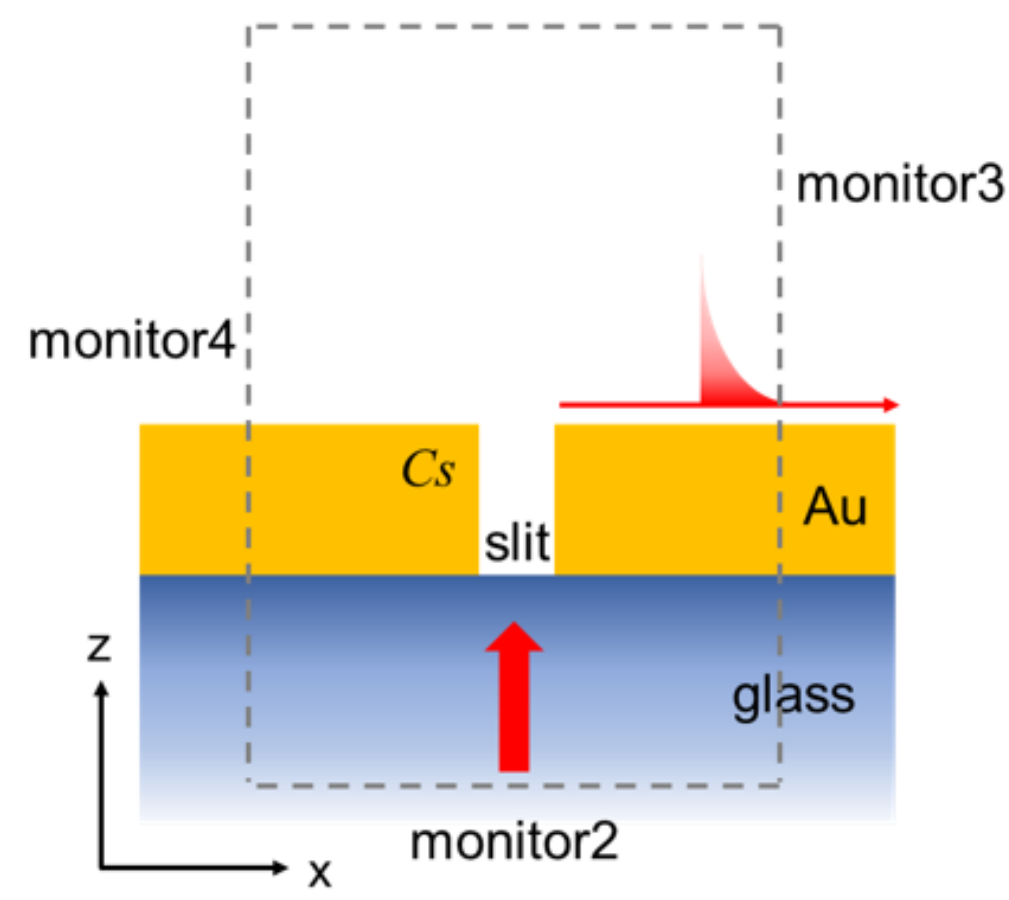

(b)

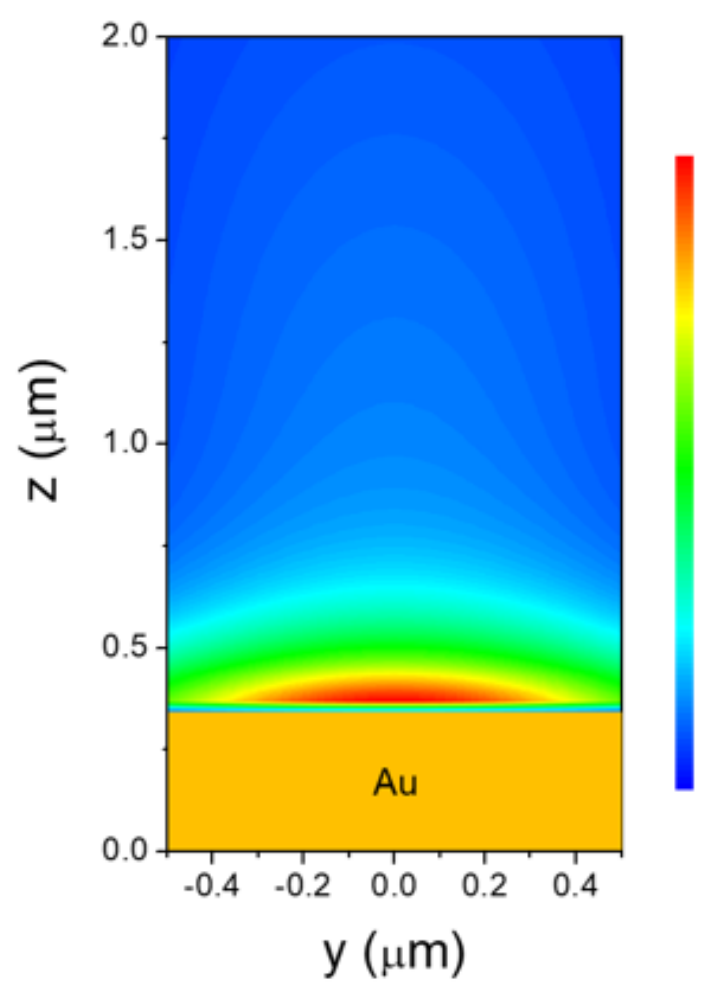

(c)

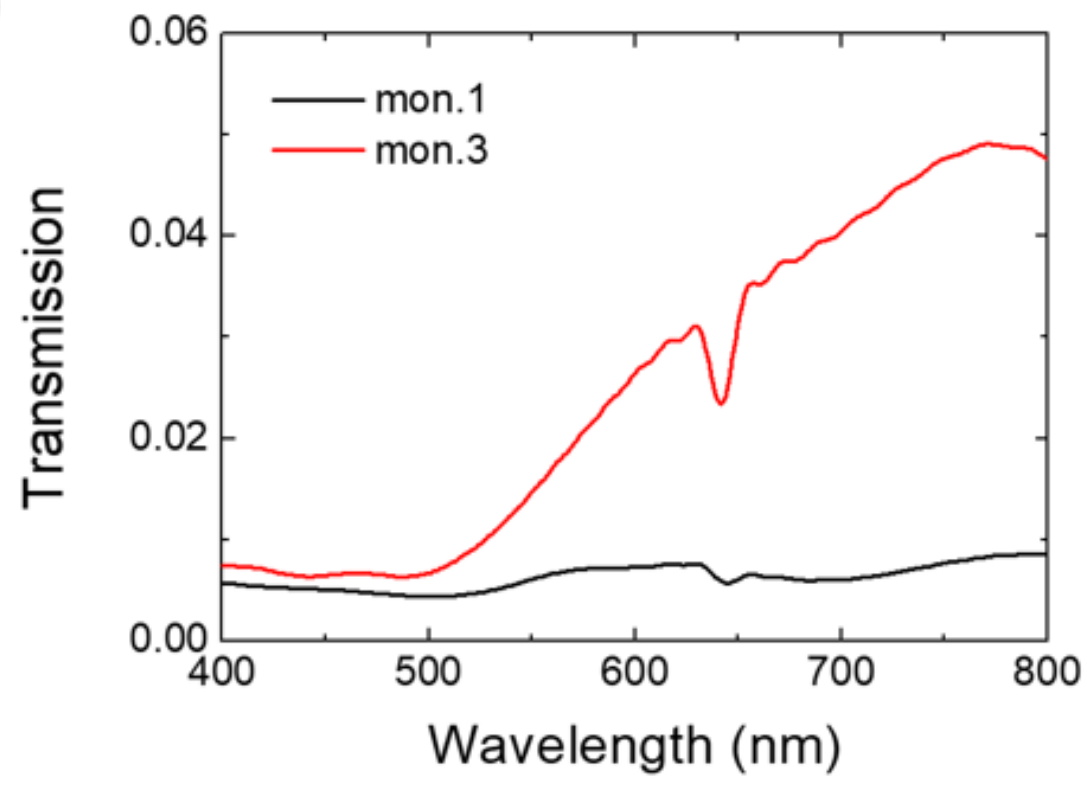

Figure 4

(a) Schematic of single slit structure used in an FDTD simulation. The dashed gray lines indicate the whole monitors used in the simulation. Monitor 1 detects the intensity transmitted through the slit and scattered by the edge of the slit. Monitor 2 detects the intensity reflected by the metal layer toward the $-\mathrm{z}$ direction. The coupling coefficient of the SPPs can be extracted from monitors 3 and 4 normal to $x$ direction. The absorption can be obtained by calculating the fields in the region enclosed by the whole 
monitors. (b) E-field intensity map obtained by monitor 3. SPP modes are formed at top surface of the metal layer. (c) Spectra plot obtained by whole monitors 